\title{
B7-H3 Blockade Decreases Macrophage Inflammatory Response and Alleviates Clinical Symptoms of Arthritis
}

Jie Yang

Suzhou Vocational Health College

\section{Yundi Guo}

Suzhou Vocational Health College

Li Gu

Suzhou Vocational Health College

Cuiping Liu

The first Affiliated Hospital of Soochow university

\section{Pingping Wu}

Suzhou Vocational Health College

\section{Min Tong}

Suzhou Vocational Health College

Jian Xiong

Suzhou Vocational Health College

Jing Sun ( $\boldsymbol{\nabla}$ jsun@szhct.edu.cn )

Suzhou Vocational Health College https://orcid.org/0000-0002-4161-6835

\section{Research article}

Keywords: rheumatoid arthritis, B7-H3, macrophages, inflammatory response

Posted Date: July 13th, 2020

DOI: https://doi.org/10.21203/rs.3.rs-41065/v1

License: (c) (1) This work is licensed under a Creative Commons Attribution 4.0 International License.

Read Full License

Version of Record: A version of this preprint was published at Immunology Letters on January 1st, 2022. See the published version at https://doi.org/10.1016/j.imlet.2021.12.004. 


\section{Abstract}

Background: The costimulatory molecule $\mathrm{B} 7-\mathrm{H} 3$ is an immunoregulatory protein that plays an important role in the course of autoimmune diseases. It is also involved in the regulation of inflammatory responses in the body. Studies have shown that B7-H3 is highly expressed in peripheral blood monocytes of rheumatoid arthritis (RA) patients and its expression is closely related to the clinical parameters of the disease. In this study we aimed to determine what the implication of high B7-H3 expression for the disease severity in human RA and mouse model of arthritis is, and whether targeting this molecule could constitute a new therapeutic approach.

Methods: We combined histopathological scoring of the joint cavity of collagen-induced arthritis (CIA) mice with immunofluorescent studies of $\mathrm{B} 7-\mathrm{H} 3$ expression on macrophages. We assessed the function of $\mathrm{B} 7-\mathrm{H} 3$ in relation with the pro-inflammatory role of macrophage through small RNA interference. Then we studied the molecular pathways liking B7-H3 with the pro-inflammatory activity of macrophages by multiplex flow cytometry, western blot and quantitative real-time PCR. The therapeutic benefit of anti-B7$\mathrm{H} 3$ blocking was probed in mouse with CIA.

Results: Histopathological and histological examination showed a positive correlation between expression of $\mathrm{B} 7-\mathrm{H} 3$ on macrophages and disease activity score, degree of destruction of the joint cavity, and pannus formation. It was also correlated with increased expression of the pro-inflammatory cytokine TNF-a in the joint cavity tissue and TNF-a level in peripheral serum. Knocking down B7-H3 expression through stable expression of small interfering RNA in human and mouse mononuclear phagocytic cell lines weakened the inflammatory response of the cells. Further molecular analyses indicated that the regulation of the inflammatory response through $\mathrm{B} 7-\mathrm{H} 3$ involved NF-KB signaling. Treatment of $\mathrm{CIA}$ mice with anti-B7-H3 reduced the clinical manifestation of arthritis and downregulated the expression of proinflammatory cytokines.

Conclusions: We confirmed increased expression of $\mathrm{B} 7-\mathrm{H} 3$ in arthritis and established a positive correlation between disease severity and expression of $\mathrm{B} 7-\mathrm{H} 3$ on macrophages. Through functional approaches in vitro and in vivo, we also demonstrated the therapeutic benefits of targeting $\mathrm{B} 7-\mathrm{H} 3$ for dampening macrophages' inflammatory responses in RA.

\section{Background}

Rheumatoid arthritis (RA) is an autoimmune disease with symmetric polyarthritis as its main clinical manifestation. It is the most common autoimmune disease, and it is characterized by massive inflammatory cell infiltration, abnormal hyperplasia of synovial tissue, and progressive destruction of articular cartilage and bone ${ }^{[1]}$. Its clinical characteristics include inflammation of the synovium of the hands, feet, wrists, knees, buttocks, and other joints. The long-lasting and repeated attacks of inflammation lead to the destruction of cartilage and bone in the joints, dysfunction of joints, and even physical disability. In addition, it can be accompanied by vascular inflammation that affects all organs of 
the body. Seventy percent of RA patients have different degrees of disability within 3 years, and therefore RA is one of the main causes of disability and labor loss ${ }^{[2]}$. RA seriously reduces human health and quality of life. However, its pathogenesis has not been fully elucidated, and it still lacks specific treatment [3].

B7-H3 (also known as CD276), which has been identified as a tumor-associated antigen in 2001, is a member of the B7 family ${ }^{[4]}$. It has been extensively studied in recent years as an important immune checkpoint molecule in the pathogenesis of tumors ${ }^{[5,6]}$. However, as an important immunomodulatory molecule, B7-H3 also plays a significant role in the pathogenesis of autoimmune diseases ${ }^{[7,8]}$. Some studies demonstrated B7-H3 as a positive costimulatory molecule and an IFN- $y$-inducer in activated human T cells. Other experiments showed that B7-H3 transfectants could down-regulate T-cell proliferation and IFN-yproduction, suggesting that it also has inhibitory effect ${ }^{[9]}$. The cellular role of B7-H3 remains controversial until now ${ }^{[10]}$. Earlier studies, including our previous study, have shown that B7-H3 is associated with autoimmune diseases, such as RA, myasthenia gravis, ankylosing spondylitis, and systemic lupus erythematosus ${ }^{[11-14]}$. The expression of B7-H3 on peripheral blood monocytes of RA patients is closely related to clinical manifestations, although the mechanism of action of B7-H3 in RA remains unclear. In the present study, we analyzed (i) the expression of $\mathrm{B} 7-\mathrm{H} 3$ on macrophages in the joint cavities of RA patients and a collagen-induced arthritis (CIA) mouse model and (ii) the correlation of B7-H3 levels with the clinicohistological evaluation of the joint cavity. We studied the effects of reduced B7-H3 expression on the cellular inflammatory response and related signaling pathways through in vitro experiments, and we treated CIA mice with anti-B7-H3 in vivo to explore the possible role of this molecule in RA treatment.

\section{Methods}

\subsection{Patients and controls}

A total of 14 RA patients (4 males and 10 females) who underwent arthroplasty at the Department of Orthopedics, the First Affiliated Hospital of Suzhou University, Jiangsu Province, China, with a mean age of $54.7 \pm 12.9$ years and a disease course of $5-30$ years were included in this study. Six patients (all males, mean age of $25.3 \pm 4.1$ years) with meniscus injury who underwent arthroscopic surgery in our hospital during the same period were selected as the control group. This study was approved by the Ethics Committee of Suzhou Health Vocational and Technology College. All patients signed written informed consent before participating.

\subsection{ClA mouse model}

The CIA mouse model was established as described in the study of Courtenay et al. ${ }^{[12]}$. Specific pathogen-free DBA/1 male mice (7-8 weeks old, weighing 18-25 g) were purchased from the Shanghai SLAC Laboratory Animal Co., Ltd. (Shanghai, China). Mice were immunized (day 0 ) intradermally at the base of the tail with $100 \mu \mathrm{g} / 100 \mu$ l bovine collagen type II (Chondrex Inc., Redmond, WA) and mixed with 
$500 \mu \mathrm{g} / 100 \mu \mathrm{l}$ of complete Freund's adjuvant (CFA) (Sigma-Aldrich).On day 21, after priming, the mice received an intradermal booster injection with the same mixture of Cll with CFA. Mice were examined visually three times per week by the double-blind method, and the different appearance of peripheral joints was observed. Morbidity of the animals was observed and evaluated according to the following scoring system: score 0 , normal; score 1, mild swelling and/or erythema; score 2, extensive swelling and/or erythema; and score 3, joint deformation and/or stiffness. Scores from all four paws were added to give the total for each animal.

\subsection{Immunohistochemistry and immunofluorescence double staining of synovial tissue in RA patients and CIA mice}

The tissues obtained from replacement surgery of RA patients were fixed in formalin and paraffinembedded. CIA mice were sacrificed a week after the onset of typical clinical symptoms including swollen or deformity ankles, the knee joints of the hind limbs were cut with bone scissors, and the surrounding tissues were removed. The joint samples were further fixed in PBS containing $10 \%$ formalin, followed by decalcification of the joints with $10 \%$ ethylenediaminetetraacetic acid (EDTA). The decalcified joint samples were embedded in paraffin, sectioned, and stained with hematoxylin and eosin (HE) and immunohistochemistry. The pathological scores of the synovial joints, including the degree of cartilage destruction of the joint, pannus formation, and the infiltration of inflammatory cells (semi-quantitative scoring system, from score 0 to 3 for each item), were determined by a pathologist. The infiltration of inflammatory cells was scored with the following system: score 0 , no inflammatory cells; score 1 , sparsely scattered infiltration of inflammatory cells; score 2 , relatively dense infiltration of inflammatory cells; and score 3 , high degree of infiltration of inflammatory cells. Pannus formation was scored as follows: score 0 , no formation; score 1, little ( $<50 \%)$ pannus formation; score $2,>50 \%$ of pannus formation; and score 3, very much pannus formation. The destruction of articular cartilage was scored as follows: score 0 , no damage; score 1, small focal damage; score 2, 50\% damage; score 3, $66.7 \%$ damage; and score 4, complete damage. The tissue sections were heated at high pressure for antigen retrieval, followed by the detection of the macrophage surface markers B7-H3 and CD68 by immunohistochemistry. Tissue sections were independently incubated with rabbit anti-B7-H3 (CD276, 1:100, Abcam) and anti-CD68 (clone KP1, Fuzhou Maixin Biology, Fujian Province, China) (80 $\mu$ l each) at $37^{\circ} \mathrm{C}$ for $1 \mathrm{~h}$, followed by washing in PBS three times ( 5 min each) and incubation with secondary antibodies $(50 \mu \mathrm{l})$ at $37^{\circ} \mathrm{C}$ for $30 \mathrm{~min}$. After three more washes in PBS, the tissue sections were stained with 3,3-diaminobenzidine (DAB) and subsequently hematoxylin, and the slides were mounted. The stained sections were observed under an Olympus optical microscope.

Fluorescence immunohistochemistry using tyramide signal amplification (TSA) technology was adopted in this study using a tyramide-coumarin conjugate kit (PerkinElmer, Waltham, MA), in accordance with the manufacturer's instructions. After adding the primary antibodies, the tyramide-coumarin working solution was added and samples were incubated for $15 \mathrm{~min}$, followed by myosin immunofluorescence labeling using an indirect immunofluorescence method ${ }^{[5]}$. Polyclonal rabbit anti-human myosin IgG (1:20) was added and incubated at $37^{\circ} \mathrm{C}$ for $1 \mathrm{~h}$, followed by three washes in PBS. The sections were then 
incubated with donkey anti-rabbit IgG-fluorescein (FITC) (1:100) at room temperature in the dark for $1 \mathrm{~h}$, followed by three washes in PBS (5 min each). Propidium iodide (PI) nuclear staining was performed by adding $1 \mu \mathrm{g} / \mathrm{ml} \mathrm{PI}$ in PBS to the tissue sections and incubating at room temperature in the dark for 3 min. After three washes in PBS at room temperature, the stained sections were observed under an AX-80 fluorescence microscope (Olympus) with three channels: the FITC signal was observed in the green channel, the coumarin signal was observed in the blue channel, and the PI signal was observed in the red channel. Images were processed using Advance SPOT software.

B7-H3 staining intensity in the cytoplasm of the inflammatory cells in the synovial tissue sections was evaluated according to the following standard: (-) unstained; $(+)<1 \%$ of nuclear staining in light yellow color; (++) $1-10 \%$ of nuclear staining or cytoplasmic staining in brown color; and $(+++)>10 \%$ nuclear staining or cytoplasmic staining in brown color. The results $(-)$ and $(+)$ were considered as negative expression, and the results (++) and (+++) were considered as positive expression of B7-H3. CD68 staining intensity was evaluated as described in the previous study of Volm et al ${ }^{[15]}$ : (-) $0-5 \%$ cytoplasmic staining; $(+) \sim 25 \%$ cytoplasmic staining; $(++) \sim 50 \%$ cytoplasmic staining; and $(+++)>50 \%$ cytoplasmic staining. The results $(-)$ and $(+)$ were considered as negative expression, and the results (++) and (+++) were considered as positive expression of CD68. The expression of the pro-inflammatory cytokine tumor necrosis factor alpha (TNF-a) was evaluated using a semi-quantitative scoring scheme based on the staining intensity, ranging from 0 (weak positive, staining intensity just exceeding background) to 3 (strong positive, dark brown staining obvious upon macroscopic inspection of the slide).

\subsection{Cell culture and viral transfection}

THP-1(monocytes from human) and RAW264.7 cells (macrophage from mouse) were purchased from Sangon Biotech Co., Ltd. (Shanghai, China). The cells were cultured in RPMI 1640 medium containing $10 \% \mathrm{FBS}$ and $100 \mathrm{U} / \mathrm{ml}$ penicillin-streptomycin at $37^{\circ} \mathrm{C}$ in a humidified incubator with $5 \% \mathrm{CO}_{2}$. The viral LV3 vector used to construct a THP-1 cell line with stable low expression of B7-H3 contained shRNA against $B 7-H 3$ and was sequenced in our previous study ${ }^{[16]}$. The sequence complementary to the shRNA against human $B 7-H 3$ used in the construction of the THP-1 stable low expression cell line started at nucleotide 1,041 of the $B 7-H 3$ sequence (LV3-hB7-H3-1041). The sequence complementary to the shRNA against mouse $B 7-H 3$ used in the construction of the RAW264.7 stable low expression cell line started at nucleotide 294 of the $B 7-H 3$ sequence (LV3-mB7-H3-294). A universal interference sequence was used as the negative control (NC). The sequences and the viral vector were synthesized by Jikai Gene Chemical Technology Co., Ltd. (Shanghai, China) (Table 1).

THP-1 and RAW264.7 cells in the exponential growth phase were seeded in 24-well plates at $5 \times 10^{4}$ cells/well and were incubated in an incubator containing $5 \% \mathrm{CO}_{2}$ at $37^{\circ} \mathrm{C}$ overnight. Culture medium was refreshed, $5 \mu \mathrm{l}$ viral stock solution was added, which was successfully packaged in the 1.2 .1 , and $5 \mu \mathrm{g} / \mathrm{ml}$ final concentration of polybrene infection/transfection reagent was added. After incubation $24 \mathrm{~h}$, the media was removed and replaced with $5 \mathrm{ml}$ of fresh growth media containing $2 \mu \mathrm{g} / \mathrm{ml}$ puromycin to 
select for stable transfectants. Single clones were picked, and the B7-H3 expression was determined using RT-PCR and Western blot analysis.

\subsection{Quantitative real-time PCR and western blot}

Total RNA was purified from THP-1 or RAW264.7 cell line using Trizol (Takara Bio Inc., Shiga, Japan).). RNA was reverse-transcribed into cDNA with an Oligo(dT) primer using a PrimeScript First cDNA synthesis kit(Takara). qRT-PCR was performed on a 7500 Real Time PCR System (Applied Biosystems) in triplicate for each target transcript using SYBRGreen PCR Master Mix (Life Technologies). Relative mRNA expression was calculated with the comparative cycle threshold method. As a calibrator, the sample with the median cycle threshold value was used and set to $100 \%$. The following primers for human B7-H3 were used: forward primer 5'-CTTGTTCGATGTTCACAGCG-3' and reverse primer 5'GCCGTAGAGCTGTCTTGGATC-3'. Mouse B7-H3 primer was: forward primer 5'TCAGTCACCATCACAGGGCA -3' and reverse primer 5'- GAGGGTTTCAGAGGCCGTAG -3'

Cell lysates (40 mg) were subjected to SDS-PAGE, then transferred electrophorectically onto polyvinyldifluoride (PVDF) membranes (Millipore, Bedford,MA). Blots were blocked with $4 \%$ BSA for $1 \mathrm{~h}$ at room temperature,then probed with primary antibodies against human B7-H3(AF1027,R\&DQ Minneapolis,MN,USA) and Mouse B7-H3(ab134161, Abcam,cambridge,MA,USA) for another hour at room temperature. After three washes, blots were subsequently incubated with the horseradish peroxidaseconjugated secondary antibodies for $1 \mathrm{~h}$ at room temperature and visualized by enhanced chemiluminescence using ImageQuant ${ }^{\mathrm{TM}}$ LAS 4000 (GE Healthcare, Pewaukee, WI).

\subsection{Establishment of an in vitro inflammation model}

THP-1 cells in the exponential growth phase were seeded in six-well plates at a concentration of $1 \times 10^{6}$ cells $/ \mathrm{ml}$. After $24 \mathrm{~h}, \mathrm{PMA}$ (at a concentration of $100 \mathrm{ng} / \mathrm{ml}$ ) was added to the cells. After $48 \mathrm{~h}$ of stimulation, the supernatants were collected and stored in a refrigerator until further analysis. The cell pellets were lysed, followed by protein extraction, and the extracted protein samples were stored at $-80^{\circ} \mathrm{C}$ until further analysis. The RAW264.7 cells were also seeded in six-well plates at a concentration of $1 \mathrm{x}$ $10^{6}$ cells $/ \mathrm{ml}$. After $24 \mathrm{~h}$, LPS (at a concentration of $100 \mathrm{ng} / \mathrm{ml}$ ) was added to stimulate the cells for $48 \mathrm{~h}$, and the supernatants and the cell pellets were collected.

\subsection{Detection of pro-inflammatory cytokine mRNAs and proteins in transfected cells and culture supernatants by quantitative RT-PCR and flow cytometric multiplex assay}

Quantitative RT-PCR were first performed to analyze the RNA expression level of pro-inflammatory cytokine. The primer sequences were as following: Mouse-TNF-a-Forward:5'-

ACAAGTCGGAGGCTTAATTACACAT-3',

Mouse-TNF-a-Reverse:5'-TTGCCATTGCACAACTCTTTTC-3';

Mouse-IL-6-Forward:5'- TCGCTCAGGGTCACAAGAAA-3', 
Mouse-IL-6-Reverse:5'- CATCAGAGGCAAGGAGGAAAAC-3';

Human-IL-6-Forward:5'- TACATCCTCGACGGCATCTC-3',

Human-IL-6- Reverse:5'- TTTCAGCCATCTTTGGAAGG-3'

Human-TNF-a- Forward:5'-CTTCTCGAACCCCGAGTGAC-3'

Human-TNF-a- Reverse:5'-ATGAGGTACAGGCCCTCTGA-3'

The serum levels of the cytokines interleukin (IL)-6, TNF-a, and IL-1 were quantified using a LegendPlex ${ }^{\text {TM }}$ Multiplex cytokine bead-based assay (BioLegend, San Diego, CA) following the manufacturer's instructions. For each reaction, $25 \mu$ of serum was diluted with $125 \mu \mathrm{l}$ deionized water. All samples were run on an Accuri C6 flow cytometer (BD Biosciences, San Diego, CA) and acquired data were analyzed using LegendPlex v7.0 software.

\subsection{NF-KB signaling}

The NF-KB Pathway Sampler Kit (catalog number: 9936, Cell Signal Technology, Danvers, MA) was used for the detection of NF-KB pathway-related proteins. Cultured cells were harvested and proteins were extracted. The levels of NF-kB p65, p-NF-кB p65, IкBa, and p-IKBa were measured by western blot.

\subsection{Treatment of CIA mice with anti-B7-H3}

$\mathrm{CIA}$ mice began to develop disease 25 days after the booster immunization. Twelve randomly selected mice were injected with monoclonal anti-B7-H3 (catalog number: 135604, Biolend) via the tail vein. Each control mouse was injected with $100 \mu \mathrm{g}$ rat IgG2a, k isotype (catalog number: 400533, Biolend) once every other day for a total of 9 days. before the beginning of the treatment and at the end of the treatment, $0.5 \mathrm{ml}$ blood sample was collected using a submandibular vein sampling needle from the submandibular vein plexus. The serum was separated and the expression levels of TNF-a, IL-6, and IL-1 $\beta$ were measured by flow cytometry. In addition, the joints of animals were evaluated and scored before and after treatment. The mice were sacrificed to dissect the joints for fixation and paraffin embedding. Tissue sectioning and staining were performed as described above, and the histological evaluation of mice was performed by a pathologist.

\subsection{Statistical analysis}

All calculations were performed with SPSS 17.0 (IBM SPSS Inc., Chicago, IL). Data are expressed as mean \pm SD. Statistical analysis was performed with the Student $t$ test and ANOVA. Correlations were evaluated by the Pearson correlation test. The $\mathrm{r}^{2}$ value was calculated to evaluate linkage disequilibrium. $P<0.05$ was considered a statistically significant difference.

\section{Results}




\subsection{B7-H3 was highly expressed on macrophages in synovial tissue of RA patients and CIA mice}

Pathological changes of synovial joint tissues were detected by HE staining. The joint tissue slices of the control group showed a clear joint cavity. The synovial cells were neatly arranged, and the noninflammatory cells infiltrated in the synovial tissue. The surface of the articular cartilage was smooth. In the experimental groups, the joint tissues showed hyperplasia of synovial lining layers, both in RA patients and CIA mice, showing more than 10 synovial lining layers. The chondrocytes were arranged disorderly, and the joint space disappeared or even merged. In addition, the tissues showed invasion of immune cells, including neutrophils, T cells, and macrophages. Tissues of some patients and mice had cartilage and synovial tissues at the joint tissues (Fig. 1A and B).

We also analyzed the expression of $\mathrm{B} 7-\mathrm{H} 3$ in the joint tissues of $16 \mathrm{RA}$ patients and $69 \mathrm{CIA}$ mice. Immunohistochemical analysis revealed that B7-H3 was expressed in synovial fibroblasts, macrophages, and other cells in the synovial tissues of RA patients and CIA mice. To further investigate whether B7-H3 was expressed in macrophages, multiple immunofluorescence analyses showed that B7-H3 and CD68 were coexpressed in the macrophages of CIA mouse joint tissues (Fig. 1C). In addition, we labeled macrophages in the tissues with anti-CD68 to analyze the infiltration of macrophages. Immunohistochemical analysis showed that the number of infiltrating macrophages in CIA mice was significantly higher than in control mice (Fig. 2A). The number of infiltrating macrophages was closely related to the joint score of the mice as evaluated by the level of synovial TNF-a (Fig. 2B) and histological analysis, including the degree of cartilage destruction and the level of angiogenesis (Fig. 2C and D).

\subsection{B7-H3 expression on macrophages reflected the clinical parameters of CIA mice and the expression of TNF-a}

Among the 113 collagen-induced mice, the CIA model was successfully established in 69 mice (Group I), and no symptom onset was observed in 44 mice (Group II). As shown in Fig. 3A, at a magnification of 200x, the numbers of B7-H3-positive macrophages were $21.49 \pm 10.06$ cells/field of view in Group I and $16.45 \pm 5.51$ cells/field of view in Group II $(P<0.0001)$; the number of B7-H3-positive macrophages in the control group was $13.5 \pm 4.64$ cells/field of view, which was significantly less than in Group II ( $P=$ $0.0004)$ and Group I $(P<0.0001)$. HE staining of the knee joint tissue sections of mouse hind limbs showed that CIA mice (i) exhibited obvious immune cell invasion and synovial inflammation at 4 and 6 weeks and (ii) had significant invasion damage of the synovium and cartilage. Using the 12-point scoring system for the evaluation of mouse joints (with a maximum score of 3 points per joint and a maximum total score of 12 points), among the 100 model mice, the highest score of the joint was 10. Immunohistochemical analysis showed that B7-H3 expression levels on macrophages were closely related to the joint score $\left(r^{2}=0.4131, P<0.0001\right.$, Fig. $\left.2 B\right)$ and to the histopathological evaluations, including cartilage destruction and pannus formation $(P<0.001)$.

The semi-quantitative immunohistochemical analysis of TNF-a expression in the joint cavity showed TNF-a was diffusely distributed in the cytoplasm of interstitial cells of joint tissues (Fig. 4A and B). TNF-a 
levels in the peripheral serum were measured by ELISA. Correlation analysis showed that higher numbers of B7-H3-positive macrophages in the joint cavity tissues were associated with stronger expression of TNF-a in joint tissues ( $P<0.0005$; Fig. 4C) and with higher levels of TNF-a in peripheral blood $(P=0.0091$; Fig. 4D).

\subsection{Stable knockdown of B7-H3 by shRNA in THP-1 and RAW264.7 macrophage cell lines dampens pro- inflammatory cytokine production upon stimulation}

To establish the in vitro inflammation cell models, $100 \mathrm{ng} / \mathrm{ml} \mathrm{PMA}$ and $100 \mathrm{ng} / \mathrm{mL}$ LPS were used to activate human THP-1 and mouse RAW264.7 macrophage cell lines[17, 18], respectively, for $48 \mathrm{~h}$. The culture medium was collected and the levels of TNF-a, IL-6, and IL-1 were measured using a multicytokine detection system.

To test the role of $\mathrm{B} 7-\mathrm{H} 3$ in inflammatory responses of human and mouse macrophages, we knocked down B7-H3 expression in THP-1 and RAW264.7 macrophages and measured their pro-inflammatory cytokine production upon stimulation. The LV3-hB7-H3-1041 viral vector prepared in our previous study was used in THP-1 cells, and the LV3-mB7-H3-294 viral vector, designed to target the sequence following nucleotide 294 in mouse B7-H3, was used in RAW264.7 cells. Real-time PCR showed than when THP-1 cells were transfected with LV3-hB7-H3-1041, the $B 7-H 3$ mRNA levels were reduced by $67.2 \%$ compared with controls (Fig. 5A). When RAW264.7 cells were transfected with LV3-mB7-H3-294, B7-H3 mRNA levels were reduced by $72.6 \%$ compared with controls. Western blot analysis confirmed that the $\mathrm{B} 7-\mathrm{H} 3$ protein levels were decreased in both cell lines (Fig. 5B-D).

After $B 7-H 3$ knockdown, the levels of TNF-a and IL-6 were significantly lower relative to the control group (Fig. 6A and $C, P<0.01$ ), while no significant changes in IL-1 levels were observed (not shown). In addition, cells were harvested and the TNF- $a$ and IL- 6 mRNA levels were measured by PCR. Both were significantly downregulated (Fig. 6B and D).

\subsection{B7-H3 regulated the inflammatory response in macrophages through the NF-kB pathway}

Because NF-KB signaling regulates the transcription and expression of various inflammatory responserelated genes, it is closely related to the occurrence and maintenance of inflammation. To further verify whether NF-KB is involved in the B7-H3-mediated regulation of inflammation in macrophages, we analyzed the levels of the NF-kB signaling-related proteins NF-kB p65, p-NF-kB p65, IкBa, and p-IкBa, in THP-1 cells after $48 \mathrm{~h}$ PMA stimulation and RAW264.7 cells after $48 \mathrm{~h}$ LPS stimulation by western blot analysis. Upon B7-H3 downregulation, the protein levels of NF-kB p65, p-NF-kB p65, IкBa, and p-IкBa were significantly decreased compared with the control group (LV-NC) (Fig. 7).

\subsection{Anti-B7-H3 alleviated the clinical manifestations and inflammation of arthritis in ClA mice}

On day 7 after symptom onset in CIA mice, monoclonal anti-B7-H3 was injected into the tail vein once every other day for 9 days. The joint scores were taken and blood was drawn from the submandibular vein before and after Ab therapy. In $\mathrm{ClA}$ mice receiving antibody treatment, the joint swelling was 
significantly reduced (Fig. 8A); the second and third toes of the mice showed the most obvious reductions (Fig. 8A). Nine ClA mice were treated with anti-B7-H3, and two of them died during treatment. Six of the remaining seven mice had a decreased joint score (Fig. 8B). After anti-B7-H3 treatment, the expression of the pro-inflammatory cytokines TNF-a, IL-1 $\beta$, and IL- 6 in five CIA mice was significantly reduced compared with before treatment (Fig. 8C). In addition, histopathological evaluation of the articular cartilage destruction, inflammatory cell infiltration, and pannus formation of the antibody-treated group showed these were significantly alleviated compared with the control group.

\section{Discussion}

Inflammation-related cells and mediators play important roles in RA. One of the most important features of RA is the sharp increase in inflammation-related cells, such as macrophages, $T$ cells, B cells, dendritic cells, and neutrophils, at the synovium of joints [19]. These inflammatory cells secrete large amounts of pro-inflammatory mediators and degradative enzymes, such as matrix metalloproteinases. These molecules mediate interactions between cells and promote the inflammatory response, which eventually leads to the destruction of the synovium, cartilage, and bone tissue [20]. Macrophages are an important class of inflammatory cells at the synovium of joints. Inactive peripheral blood monocytes mature and infiltrate the inflamed tissues, where they differentiate into macrophages upon stimulation and regulation by pro-inflammatory cytokines [21, 22]. In turn, they interact with activated T cells, fibroblasts, epithelial cells, and NK cells in the joints by releasing pro-inflammatory cytokines, such as TNF-a and IL-1, chemokines, and macrophage migration inhibitory factors [23]. In the present study, we observed significantly more infiltrated macrophages in the joint tissues of RA patients and CIA mice than in healthy individuals and control mice. In addition, the secretion of inflammatory cytokines in the tissues was high. The degree of macrophage infiltration was positively correlated with the secretion of inflammatory cytokines, such as TNF-a, and higher degrees of cartilage destruction and pannus formation were associated with higher degrees of macrophage infiltration.

In general, the protein levels of B7-H3 are relatively limited compared with the mRNA levels ${ }^{[4]}$. Except for malignant tumor tissues, different degrees of $\mathrm{B} 7-\mathrm{H} 3$ expression have only been detected on dendritic cells and macrophages in tissues and activated peripheral blood monocytes in vitro ${ }^{[24,25]}$. In a previous study we showed that the expression of $\mathrm{B} 7-\mathrm{H} 3$ on the surface of $\mathrm{CD} 14+$ monocytes in peripheral blood of RA patients is significantly higher than in healthy individuals, and is positively correlated with the clinical pathology and parameters, including the number of swollen joints, the DSA28 score index, the duration of disease, the erythrocyte sedimentation rate, and the levels of rheumatoid factors [14]. Another study has shown that B7-H3 is expressed in the synovial tissues of RA patients, CD68 + macrophages, synovial fibroblasts, CD31 + endothelial cells, and CK18 + epithelial cells [26]. In the present study, we analyzed B7$\mathrm{H} 3$ expression on macrophages of synovial tissues from joints of RA patients and CIA mice by immunohistochemical analysis and immunofluorescence costaining, and observed that B7-H3 expression in these cells was significantly higher than on the macrophages of normal synovial tissues. Furthermore, we demonstrated that B7-H3 expression levels were positively correlated with the clinical 
and histological evaluations, including joint score, cartilage destruction, and pannus formation, and were weakly correlated with the secretion of inflammatory cytokines. Most studies have shown that B7-H3 has a co-inhibitory role on immune response, contributing to tumor cell immune evasion Targeting B7-H3 in cancer treatment can reduce cell proliferation, progression, and metastasis, which may ultimately lead to improved therapeutic options and better clinical outcomes. Our results novelty indicated B7-H3 might act a co-stimulate role in immune response and related with inflammation-related autoimmune disorders.

What role does high B7-H3 expression levels on macrophages in joint tissue play in RA? B7-H3 may be related to the inflammatory regulation of macrophages. A study has shown that $\mathrm{B} 7-\mathrm{H} 3$ significantly enhances the toll-like receptor (TLR)-4 agonist LPS and the TLR-2 agonist bacterial lipoprotein to stimulate the production of pro-inflammatory cytokines in monocytes and macrophages, regulating the inflammatory response; MIH35 (an anti-B7-H3 antibody) significantly reduces the release of proinflammatory cytokines [27]. Chen et al. have shown that B7-H3 activates TLR-2 downstream signaling pathways, NF-KB p65, and MAPK p38 in mice infected with Streptococcus pneumoniae. Inhibitors of the above pathways weaken the pro-inflammatory responses and reduce the production of pro-inflammatory cytokines and chemokines [28]. To further explore the effects of high B7-H3 expression on macrophages, we constructed THP-1 cells (human mononuclear phagocytes) and RAW264.7 cells (mouse macrophages) with stable low $\mathrm{B} 7-\mathrm{H} 3$ expression, and showed that these stable $B 7-H 3$ knockdown cell lines had reduced secretion of pro-inflammatory cytokines, including IL-6 and TNF-a, under an inflammatory environment. In addition, signaling pathway analysis showed that NF-KB signaling was inhibited. We confirmed that B7-H3 may enhance the ability of macrophages to regulate inflammation through the NF-KB signaling pathway and participate in RA progression.

To verify our hypothesis, we treated CIA mice with anti-B7-H3. Moreover, the levels of the proinflammatory cytokines IL-6 and TNF-a in the peripheral blood were significantly lower, and the expression of inflammatory cytokines, the degree of inflammatory cell infiltration, and the degree of bone and joint destruction were significantly reduced. Based on our findings, anti-B7-H3 treatment may reduce inflammation and mitigate RA symptoms and damage.

\section{Conclusions}

In this study, we have shown that high expression levels of B7-H3 in macrophages in RA tissues enhance the ability of macrophages to promote the inflammatory response. Blockade with anti-B7-H3 reduced the inflammatory response, suggesting that $\mathrm{B} 7-\mathrm{H} 3$ may be used as a drug target for the treatment of RA.

\section{Abbreviations}

Ab: Antibody; bp: base pair; CIA: Collagen-induced arthritis; EDTA: ethylenediaminetetraacetic acid; H \& E: Hematoxylin and eosin; HRP: Horseradish peroxidase; Ig: Immunoglobulin; IL: Interleukin; mAb: Monoclonal antibody; PBS: Phosphate-buffered saline; PMA: Phorbol-12-myristate-13-acetate; LPS: 
Lipopolysaccharides; TLR: toll-like receptor; RT-PCR: Real-time polymerase chain reaction; RA: rheumatoid arthritis; NC:negative control.

\section{Declarations}

\section{Ethics approval and consent to participate}

The study was approved by the local ethics committee and conducted according to the guidelines of the Declaration of Helsinki. Written informed consent was obtained from all patients (Suzhou Vocational Health College Medical Faculty Ethics Approval No. 004).

\section{Consent for publication}

Not applicable

\section{Availability of data and materials}

The data that support the findings of this study are available on reasonable request from the corresponding author.

\section{Competing interests}

The authors declare that they have no competing interests.

\section{Funding}

This work was supported by Grants from Jiangsu Province engineering Research Center (Medical Diagnostic AntibodyNO:2018026) and the Jiangsu Province University Outstanding Science and Technology Innovation Team (Grant No. 2015023), Jiangsu Provincial Medical Talent (Sunjing).The funders had no role in the study design, data collection and analysis, decision to publish, or preparation of the manuscript.

\section{Authors' contributions}

Research was designed by Jing sun. Patient samples and data were provided by Cuiping Liu and Min Tong. Cells experiments in Vitro and animal experiments in Vivo were performed by Jie Yang and Jian Xiong. All histochemistry preparations and analysis were performed by Yundi Guo and Li Gu . Jing Sun had full access to all of the data in the study and takes responsibility for the integrity of the data and the accuracy of the data analysis. All authors were involved in drafting the manuscript or revising it critically for important intellectual content. All authors read and approved the final manuscript.

\section{Acknowledgements}

We thank Xiaoning Li for his technical support. 


\section{References}

1. Yu C, Li M, Duan X, Fang Y, Li Q, Wu R et al. Chinese registry of rheumatoid arthritis (CREDIT): I. Introduction and prevalence of remission in Chinese patients with rheumatoid arthritis. Clin Exp Rheumatol. 2018;36(5):836-40. doi:12256 [pii].

2. Qian J, Xu L, Sun X, Wang Y, Xuan W, Zhang Q et al. Adiponectin aggravates bone erosion by promoting osteopontin production in synovial tissue of rheumatoid arthritis. Arthritis Res Ther. 2018;20(1):26. doi:10.1186/s13075-018-1526-y 10.1186/s13075-018-1526-y [pii].

3. Zamanpoor M. The Genetic Pathogenesis, Diagnosis and Therapeutic insight of Rheumatoid Arthritis. Clin Genet. 2018. doi:10.1111/cge.13498.

4. Chapoval Al, Ni J, Lau JS, Wilcox RA, Flies DB, Liu D et al. B7-H3: a costimulatory molecule for T cell activation and IFN-gamma production. Nat Immunol. 2001;2(3):269-74. doi:10.1038/85339.

5. Zhai L, Ladomersky E, Lenzen A, Nguyen B, Patel R, Lauing KL et al. ID01 in cancer: a Gemini of immune checkpoints. Cell Mol Immunol. 2018. doi:10.1038/cmi.2017.143 cmi2017143 [pii].

6. Flem-Karlsen K, Fodstad O, Tan M, Nunes-Xavier CE. B7-H3 in Cancer - Beyond Immune Regulation. Trends Cancer. 2018;4(6):401-4. doi:S2405-8033(18)30088-8 [pii] 10.1016/j.trecan.2018.03.010.

7. Chen Y, Guan SY, Deng J, Yang H, Xu W, Xu S et al. B7-H3: A promising therapeutic target for autoimmune diseases. Cell Immunol. 2020:104077. doi:S0008-8749(19)30512-X [pii] 10.1016/j.cellimm.2020.104077.

8. Chen Y, Guan SY, Deng J, Yang H, Xu W, Xu S et al. B7-H3: A promising therapeutic target for autoimmune diseases. Cell Immunol. 2020;352:104077. doi:S0008-8749(19)30512-X [pii] 10.1016/j.cellimm.2020.104077.

9. Sun X, Yu Y, Ma L, Xue X, Gao Z, Ma J et al. T cell cytotoxicity toward hematologic malignancy via B7-H3 targeting. Invest New Drugs. 2020;38(3):722-32. doi:10.1007/s10637-019-00819-y 10.1007/s10637-019-00819-y [pii].

10. Li G, Quan Y, Che F, Wang L. B7-H3 in tumors: friend or foe for tumor immunity? Cancer Chemother Pharmacol. 2018;81(2):245-53. doi:10.1007/s00280-017-3508-1 10.1007/s00280-017-3508-1 [pii].

11. Sun J, Lai H, Shen D, Wu P, Yang J, Sun Z et al. Reduced sB7-H3 Expression in the Peripheral Blood of Systemic Lupus Erythematosus Patients. J Immunol Res. 2017;2017:5728512. doi:10.1155/2017/5728512.

12. Jiang J, Liu C, Zhang G, Gao L, Chen Y, Zhu R et al. Enhancement of membrane B7-H3 costimulatory molecule but reduction of its soluble form in multiple sclerosis. J Clin Immunol. 2013;33(1):118-26. doi:10.1007/s10875-012-9800-2.

13. Yan R, Hu Y, Yu P, Wen H, Feng P, Yang S. Reduced serum B7-H3 levels in patients with ankylosing spondylitis. Inflammation. 2015;38(3):1322-8. doi:10.1007/s10753-014-0103-z.

14. Sun J, Liu C, Gao L, Guo Y, Zhang Y, Wu P et al. Correlation between B7-H3 expression and rheumatoid arthritis: A new polymorphism haplotype is associated with increased disease risk. Clin Immunol. 2015;159(1):23-32. doi:10.1016/j.clim.2015.04.012 S1521-6616(15)00142-4 [pii]. 
15. Volm M, Koomagi R, Mattern J. Prognostic value of vascular endothelial growth factor and its receptor Flt-1 in squamous cell lung cancer. Int J Cancer. 1997;74(1):64-8. doi:10.1002/(SICI)10970215(19970220)74:1<64::AID-IJC11>3.0.C0;2-Ipii] 10.1002/(sici)10970215(19970220)74:1<64::aid-ijc11>3.0.co;2-i.

16. Lai H, Sun Z, Yang J, Wu P, Guo Y, Sun J. B7-H3 modulates endothelial cell angiogenesis through the VEGF cytokine. Immunol Res. 2019;67(2-3):202-11. doi:10.1007/s12026-019-09084-w 10.1007/s12026-019-09084-w [pii].

17. Hwang JH, Kim KJ, Ryu SJ, Lee BY. Caffeine prevents LPS-induced inflammatory responses in RAW264.7 cells and zebrafish. Chem Biol Interact. 2016;248:1-7. doi:10.1016/j.cbi.2016.01.020 S0009-2797(16)30020-5 [pii].

18. Tedesco S, De Majo F, Kim J, Trenti A, Trevisi L, Fadini GP et al. Convenience versus Biological Significance: Are PMA-Differentiated THP-1 Cells a Reliable Substitute for Blood-Derived Macrophages When Studying in Vitro Polarization? Front Pharmacol. 2018;9:71. doi:10.3389/fphar.2018.00071.

19. Chen Z, Bozec A, Ramming A, Schett G. Anti-inflammatory and immune-regulatory cytokines in rheumatoid arthritis. Nat Rev Rheumatol. 2019;15(1):9-17. doi:10.1038/s41584-018-0109-2 10.1038/s41584-018-0109-2 [pii].

20. Peng $H$, Herzog EL. Fibrocytes: emerging effector cells in chronic inflammation. Curr Opin Pharmacol. 2012;12(4):491-6. doi:10.1016/j.coph.2012.03.002 S1471-4892(12)00044-6 [pii].

21. Koga T, Kawashiri SY, Migita K, Sato S, Umeda M, Fukui S et al. Comparison of serum inflammatory cytokine concentrations in familial Mediterranean fever and rheumatoid arthritis patients. Scand $\mathrm{J}$ Rheumatol. 2018;47(4):331-3. doi:10.1080/03009742.2017.1363281.

22. Mateen S, Moin S, Shahzad S, Khan AQ. Level of inflammatory cytokines in rheumatoid arthritis patients: Correlation with 25-hydroxy vitamin D and reactive oxygen species. PLoS One. 2017;12(6):e0178879. doi:10.1371/journal.pone.0178879 PONE-D-17-05422 [pii].

23. Jin S, Li M, Fang Y, Li Q, Liu J, Duan X et al. Chinese Registry of rheumatoid arthritis (CREDIT): II. prevalence and risk factors of major comorbidities in Chinese patients with rheumatoid arthritis. Arthritis Res Ther. 2017;19(1):251. doi:10.1186/s13075-017-1457-z 10.1186/s13075-017-1457-z [pii].

24. Wang Z, Zhang C, Liu X, Li G, Liu S, Sun L et al. Genetic and clinical characterization of B7-H3 (CD276) expression and epigenetic regulation in diffuse brain glioma. Cancer Sci. 2018;109(9):2697705. doi:10.1111/cas.13744.

25. Xu JC, Li M, Ma Y, Shi CL, Zhu XY, Song HF et al. The Expression of B7-H3 in Circulating CD4+CD25high T Cells, Circulating CD14+ Monocytes and Plasma during Hepatitis B Virus Infection Progression. Clin Lab. 2017;63(3):617-23. doi:10.7754/Clin.Lab.2016.160811.

26. Yoon BR, Chung YH, Yoo SJ, Kawara K, Kim J, Yoo IS et al. Preferential Induction of the T Cell Auxiliary Signaling Molecule B7-H3 on Synovial Monocytes in Rheumatoid Arthritis. J Biol Chem. 2016;291(8):4048-57. doi:10.1074/jbc.M115.680298 M115.680298 [pii]. 
27. Zhang G, Wang J, Kelly J, Gu G, Hou J, Zhou Y et al. B7-H3 augments the inflammatory response and is associated with human sepsis. J Immunol. 2010;185(6):3677-84. doi:10.4049/jimmunol.0904020 jimmunol.0904020 [pii].

28. Chen X, Bai Y, Cui W, Wang Z, Zhang G, Xu Y et al. Effects of B7-H3 on the inflammatory response and expression of MMP-9 in mice with pneumococcal meningitis. J Mol Neurosci. 2013;50(1):14653. doi:10.1007/s12031-012-9885-3.

\section{Figures}

A

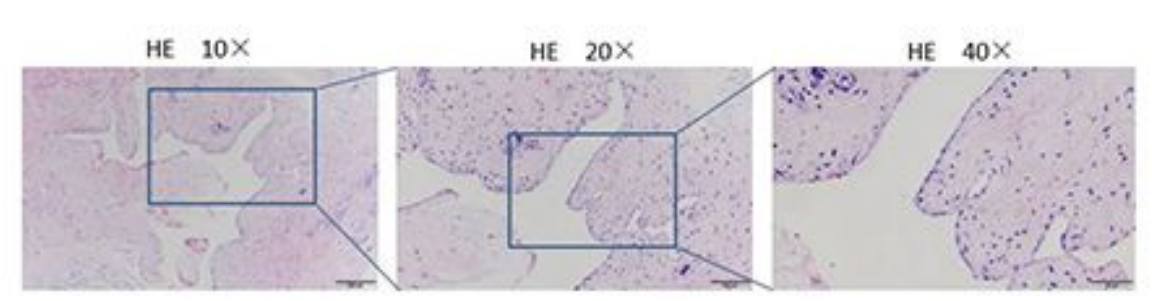

$\mathrm{HC}$
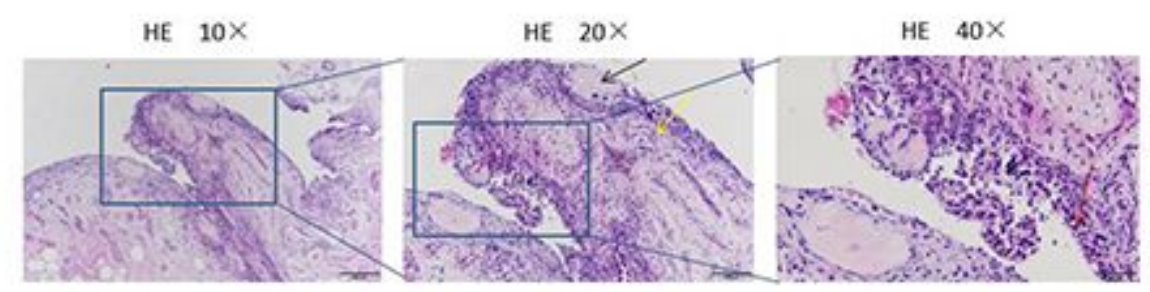

$\operatorname{CD68} 40 \times$

B7. $\mathrm{H3} 40 \times$
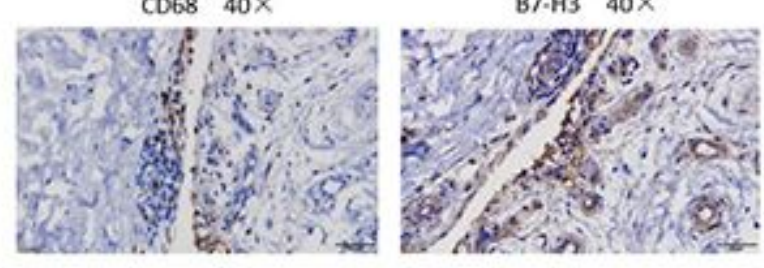

B

HE $10 \times$

HE $20 \times$

HE $40 \times$

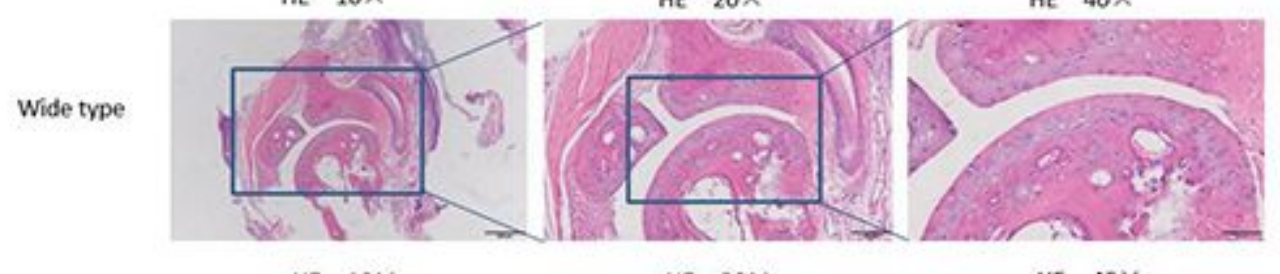

CIA

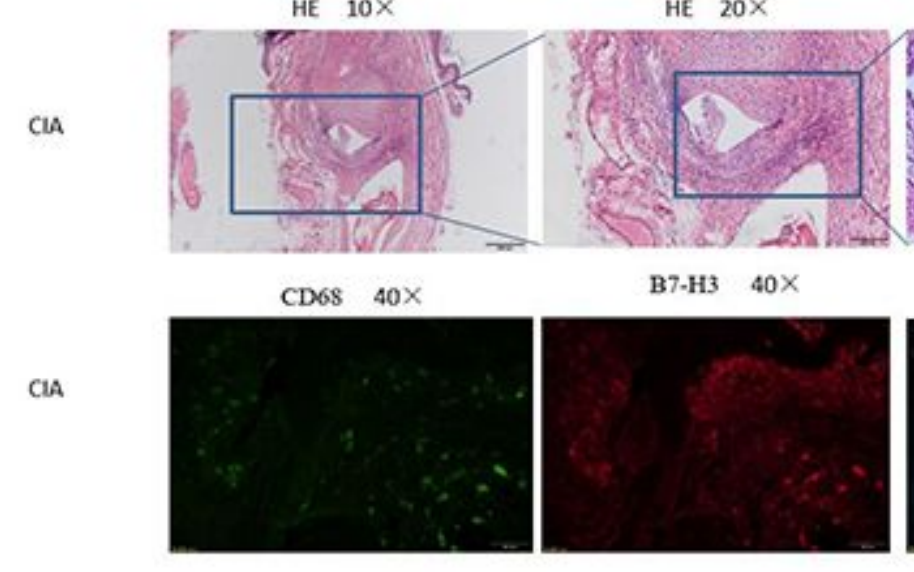

HE $20 X$

HE $40 X$

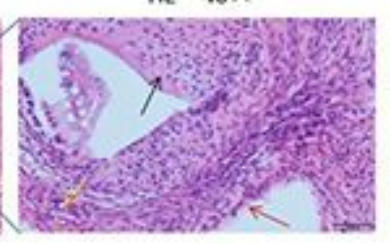

CD68/B7-H3 $40 \mathrm{X}$

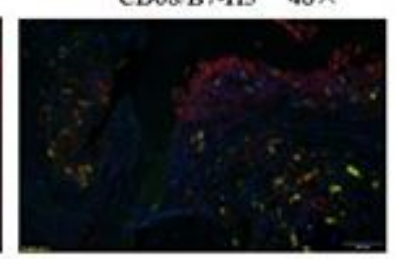


B7-H3 was highly expressed in the synovial tissues of RA patients and CIA mice. (A) HE staining and antiCD68, anti-B7-H3 staining of the synovial tissue of Heathy control donors and RA patients; the cartilage destruction is identified by the black arrow head and synovial lining thickness by the red bracket, and the inflammation and erosion/pannus were indicated by yellow arrow head. (B) HE staining of the synovial tissue of wide-type mice and CIA mice; $n=12$ in RA group and $n=69$ in CIA group. Arrow heads show the cartilage destruction, lining thickness and pannus. Coexpression of $\mathrm{CD} 68$ and $\mathrm{B} 7-\mathrm{H} 3$ in the synovial tissues of CIA mice. Green, CD68 (macrophages); red, B7-H3; yellow, blue, DAPI nuclear staining. All images represent CIA mice with approximate mean summed paw score. $\mathrm{n}=69$ in $\mathrm{CIA}$ group.

A

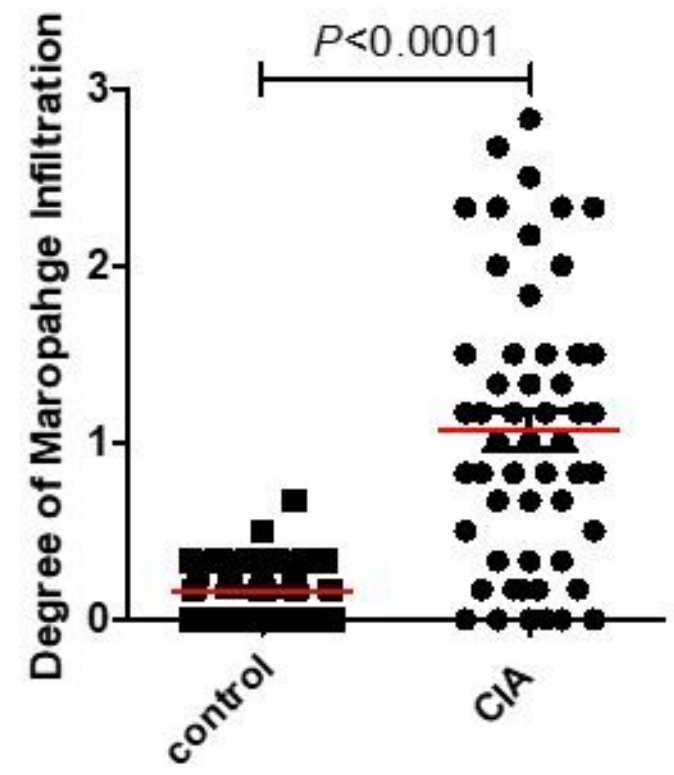

C

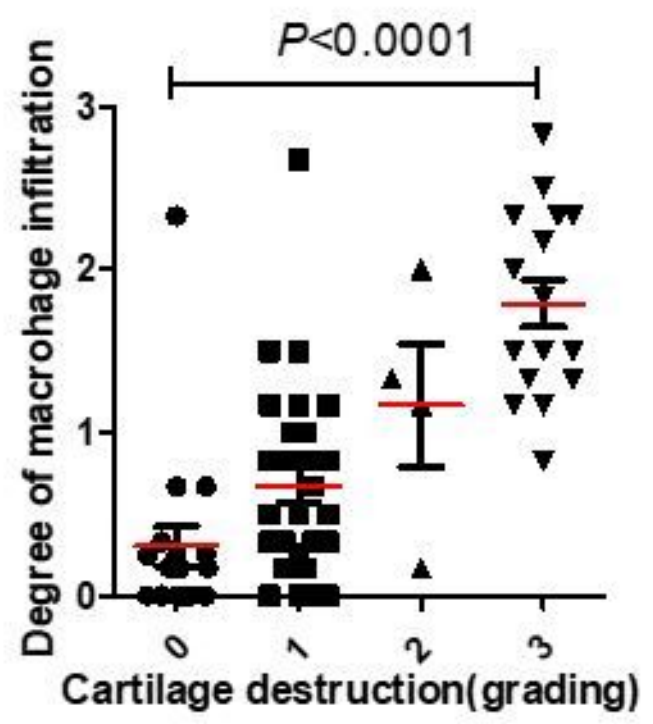

$\mathrm{B}$

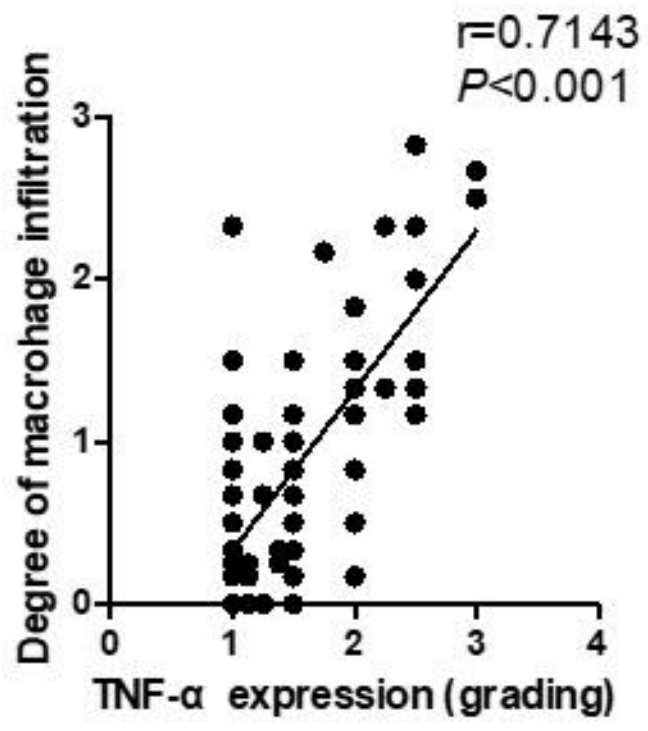

D

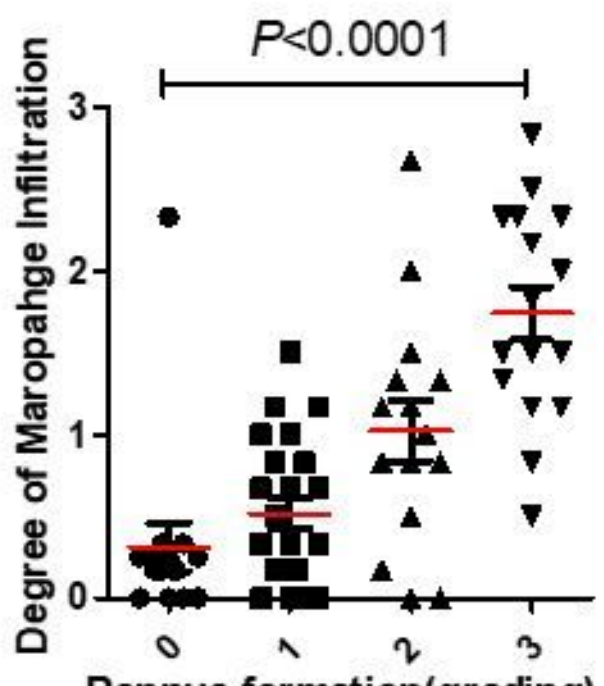

Pannus formation(grading)

Figure 2

The relationship between infiltration of macrophages in synovial joints in CIA mice and the clinical and histological evaluation of the disease. (A) The infiltration of macrophages in CIA mice $(n=69)$ was significantly increased compared with control mice $(n=40)$. (B) The degree of macrophage infiltration was closely related to the expression of TNF-a in the synovium of CIA mice $(n=69)(P<0.001)$. (C) The degree 
of macrophage infiltration was significantly associated with the destruction of synovial cartilage $(\mathrm{P}<$ $0.001) . n=19$ in grading 0,29 in grading1, 4 in grading2, 16 in grading 3 . (D) The degree of macrophage infiltration was significantly associated with pannus formation $(P<0.001) . n=15$ in grading 0,23 in grading1, 15 in grading2, 16 in grading 3.
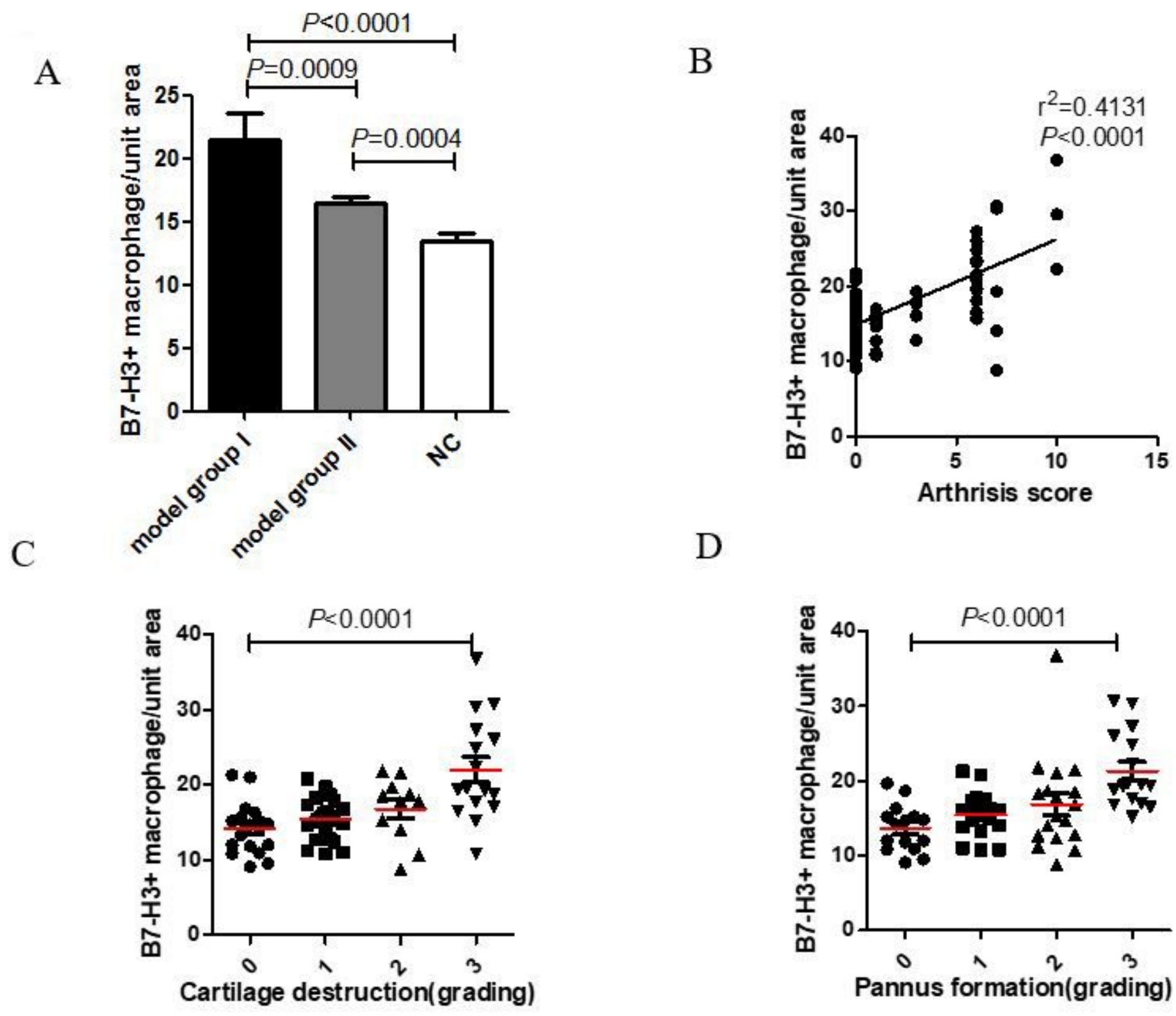

$\mathrm{D}$

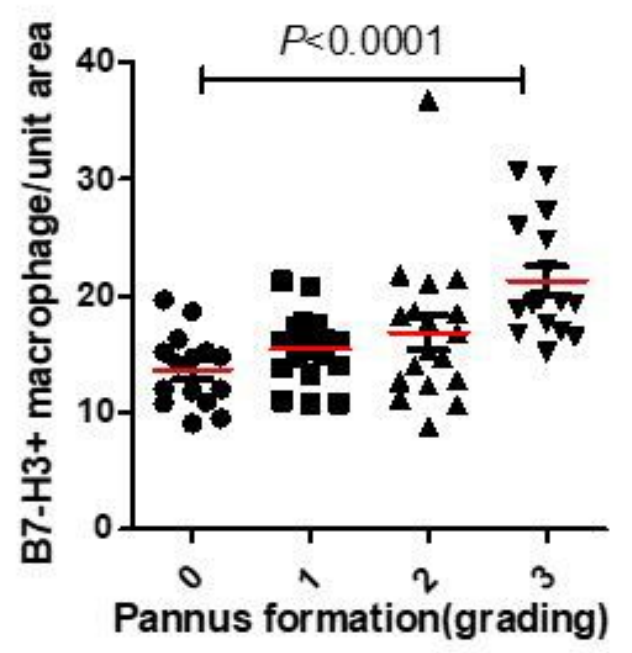

Figure 3

The relationship between the expression of $\mathrm{B} 7-\mathrm{H} 3$ on macrophages in synovial joint tissues and the clinical and histological evaluation of the disease. (A) Collagen-treated mice in which the RA model was successfully established (Group I) had higher B7-H3 expression levels than collagen-treated mice without symptom onset (Group II). Groups I and II exhibited higher B7-H3 expression than the non-induced control group (NC). $n=69$ in Group I. $n=44$ in group II. (B) The expression of B7-H3 on macrophages was closely related to the clinical score of the CIA mice joints $(P<0.0001)$. (C) The expression of $B 7-\mathrm{H} 3$ on 
macrophages was related to the degree of destruction of articular cartilages. (D) The expression of B7-H3 on macrophages was related to pannus formation. $n=69$ in (B), (C) and (D) group.

TNF- $\alpha 10 \times$

TNF- $\alpha 20 \times$

TNF- $\alpha 40 \times$

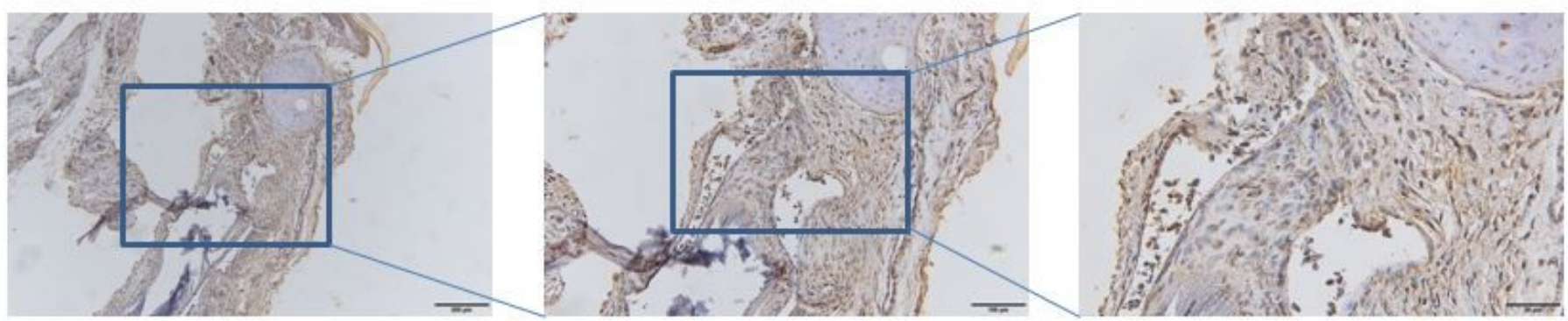

$\mathrm{C}$

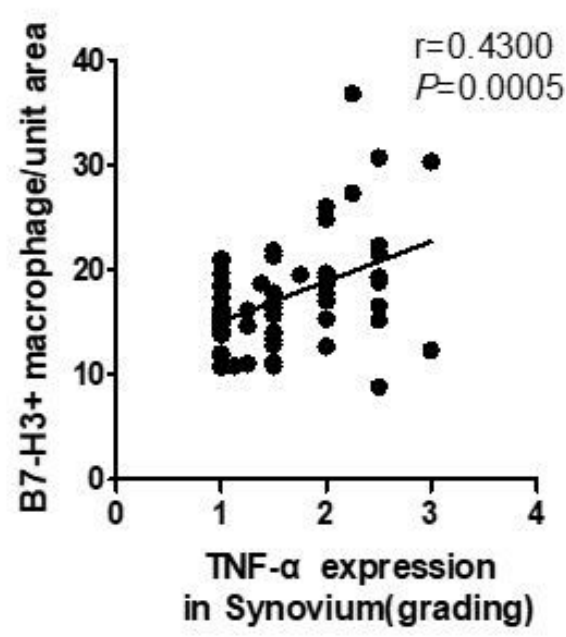

$\mathrm{D}$

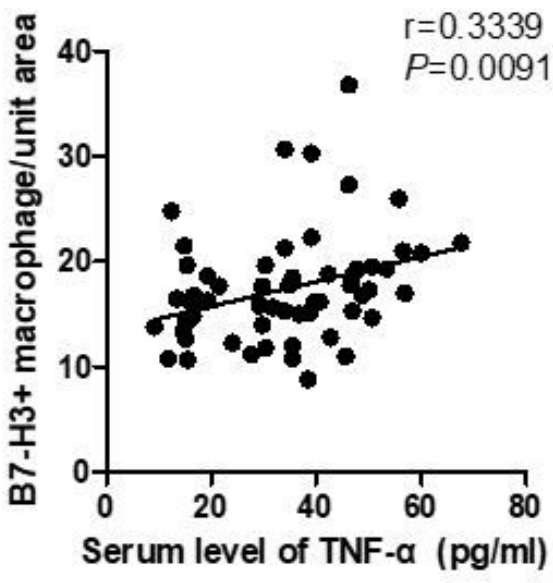

TNF- $\alpha \times 40$

\section{Figure 4}

The relationship between the expression of B7-H3 on macrophages and TNF- $\mathrm{a}$ expression in synovial joint tissues. (A \& B) Representative images of TNF-a immunohistochemical staining in joint tissue under light microscopy at 10x and 40x magnifications. (C) Expression levels of TNF-a in joint tissue were closely related to the expression of $B 7-H 3$ on macrophages $(P=0.0005)$. (D) The TNF-a serum levels in the peripheral blood of CIA mice were closely related to the expression of $\mathrm{B} 7-\mathrm{H} 3$ on macrophages $(\mathrm{P}=$ $0.0005) . n=69$ in CIA group. 
A

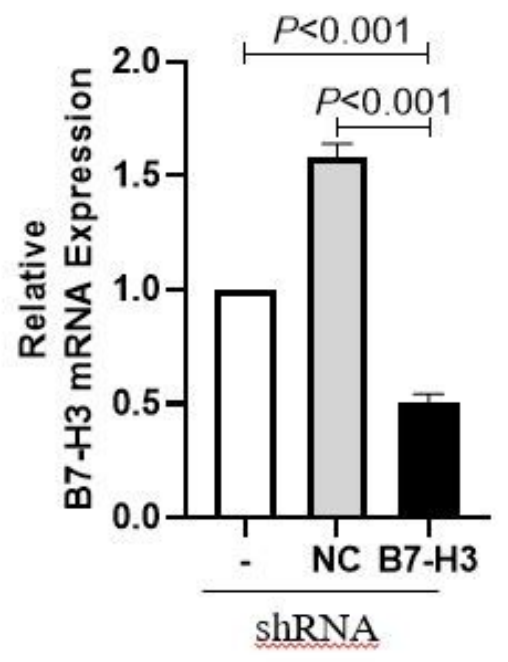

$\mathrm{C}$

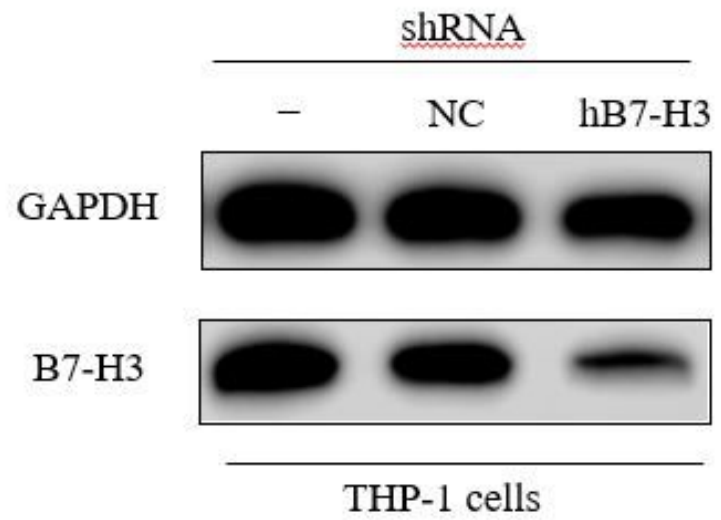

B

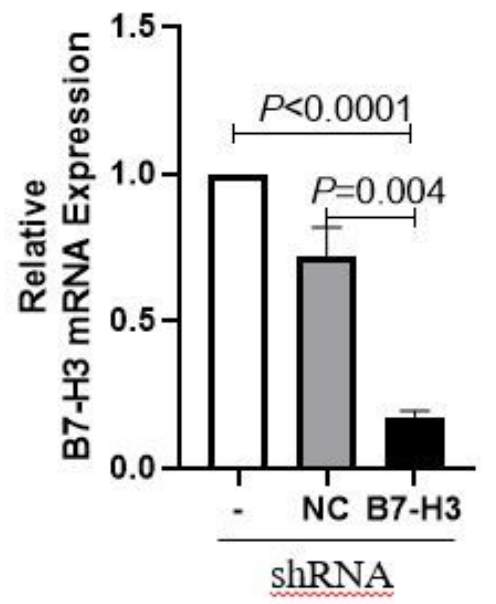

$\mathrm{D}$

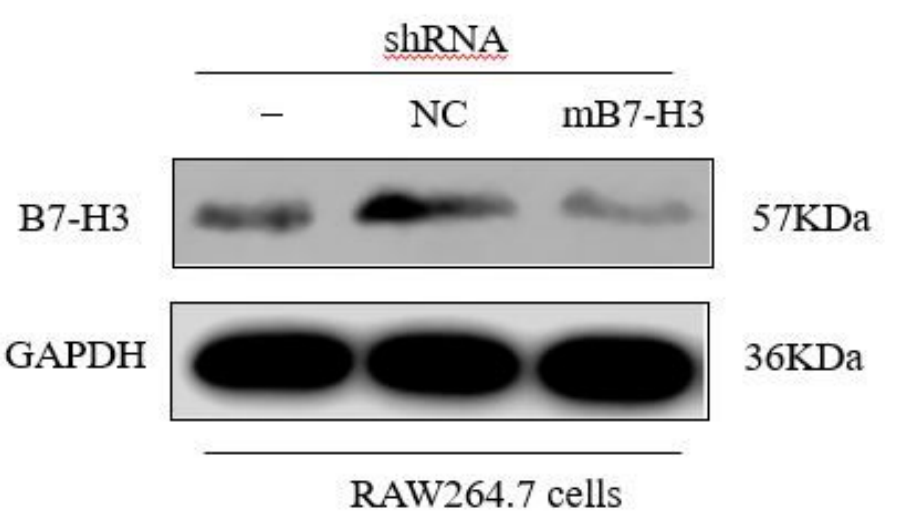

\section{Figure 5}

Construction of a human macrophage THP-1 cell line and a mouse macrophage RAW264.7 cell line with low B7-H3 expression. (A) Quantitation of the effects of LV3-hB7-H3-1041 on the mRNA levels of B7-H3 by PCR (THP-1/hB7-H3-1041) compared to non-transfected cells (THP-1) and cells transfected with control shRNA (THP-1/NC). (B) Quantitation of the effects of LV3-mB7-H3-294 on the mRNA levels of B7H3 by PCR (RAW264.7/mB7-H3-294) compared to non-transfected cells (RAW264.7) and cells transfected with control shRNA (RAW264.7/NC). (C) Western blot analysis confirmed low B7-H3 protein expression in THP-1 cells transfected with LV3-hB7-H3-1041. (D) Western blot analysis confirmed low B7-H3 protein expression in RAW264.7 cells transfected with LV3-mB7-H3-294. Data are presented as means \pm SD from three independent experiments.Construction of a human macrophage THP-1 cell line and a mouse macrophage RAW264.7 cell line with low B7-H3 expression. (A) Quantitation of the effects of LV3-hB7-H31041 on the mRNA levels of B7-H3 by PCR (THP-1/hB7-H3-1041) compared to non-transfected cells (THP-1) and cells transfected with control shRNA (THP-1/NC). (B) Quantitation of the effects of LV3-mB7H3-294 on the mRNA levels of B7-H3 by PCR (RAW264.7/mB7-H3-294) compared to non-transfected cells (RAW264.7) and cells transfected with control shRNA (RAW264.7/NC). (C) Western blot analysis confirmed low B7-H3 protein expression in THP-1 cells transfected with LV3-hB7-H3-1041. (D) Western 
blot analysis confirmed low B7-H3 protein expression in RAW264.7 cells transfected with LV3-mB7-H3294. Data are presented as means \pm SD from three independent experiments.

THP-1

A

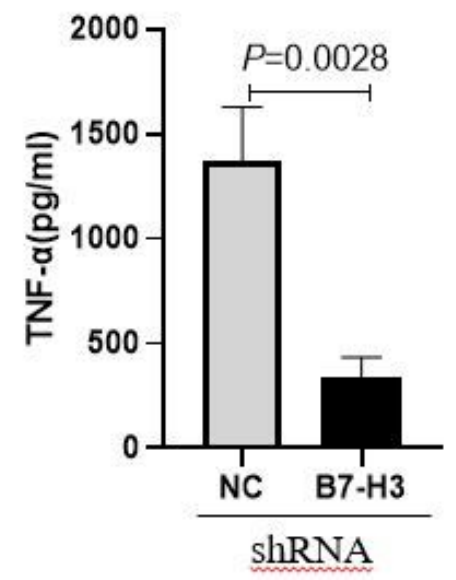

$\mathrm{C}$

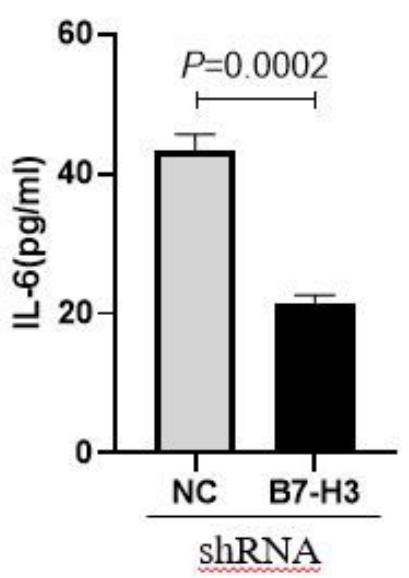

Raw 264.7

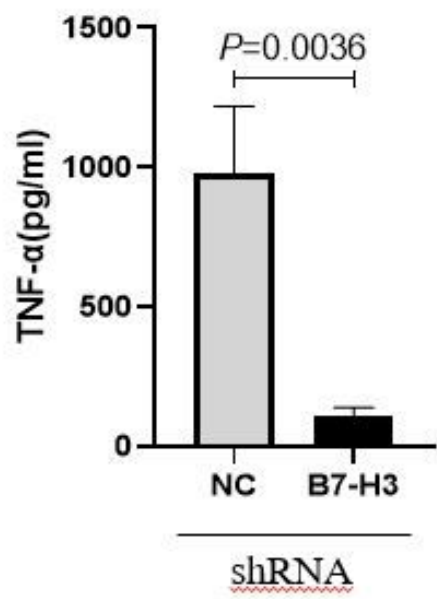

B

THP-1 cells

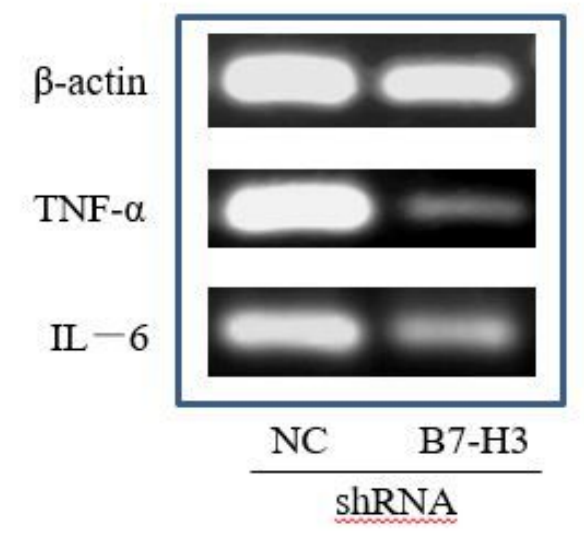

$\mathrm{D}$

THP-1

B
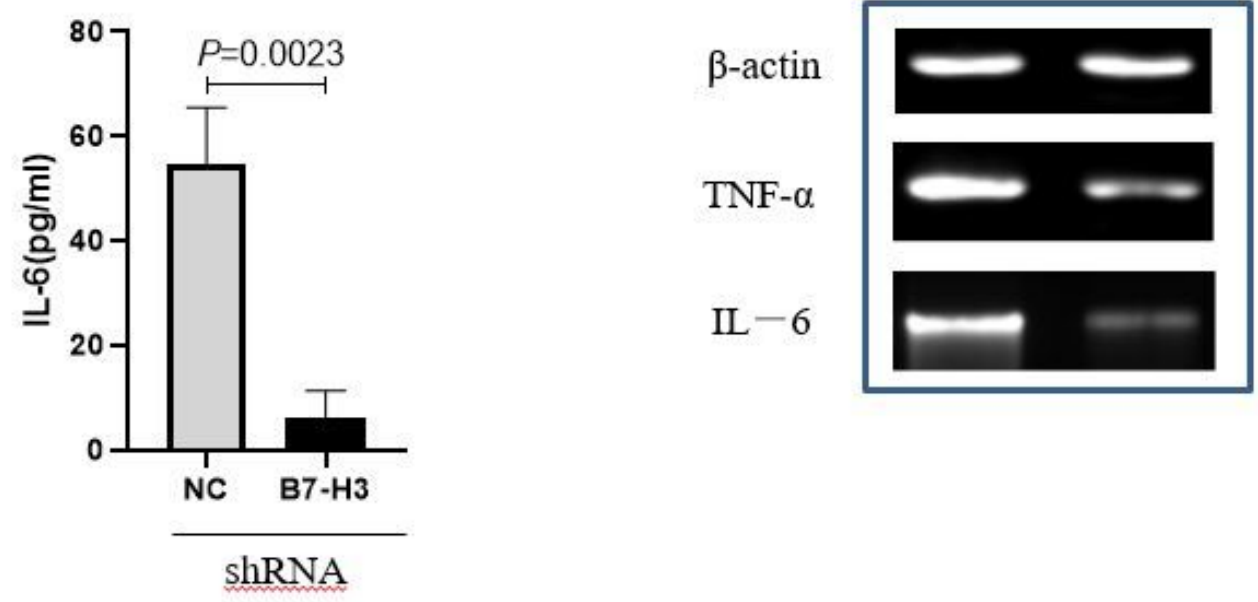

Figure 6

Detection of TNF- $a$ and IL-6. (A) The serum levels of TNF- $a$ and IL- 6 were significantly reduced after downregulating B7-H3 expression in THP-1 cells. (B) PCR analyses of TNF-a and IL- 6 mRNA levels in THP-1 cells transfected with LV3-hB7-H3-1041 and control cells. (C) The serum levels of TNF-a and IL-6 were significantly reduced after downregulating B7-H3 expression in RAW264.7 cells. (D) PCR analyses of TNF-a and IL-6 mRNA levels in RAW264.7 cells transfected with LV3-mB7-H3-294 and control cells. Data are presented as means \pm SD from four independent experiments. 
A

THP-1 cells

$$
\begin{aligned}
& \text { NF-kB p65 } \\
& \text { p-NF-kB p65 }
\end{aligned}
$$

$I \mathrm{kB}$ a

$p-1 k B a$

GAPDH
B

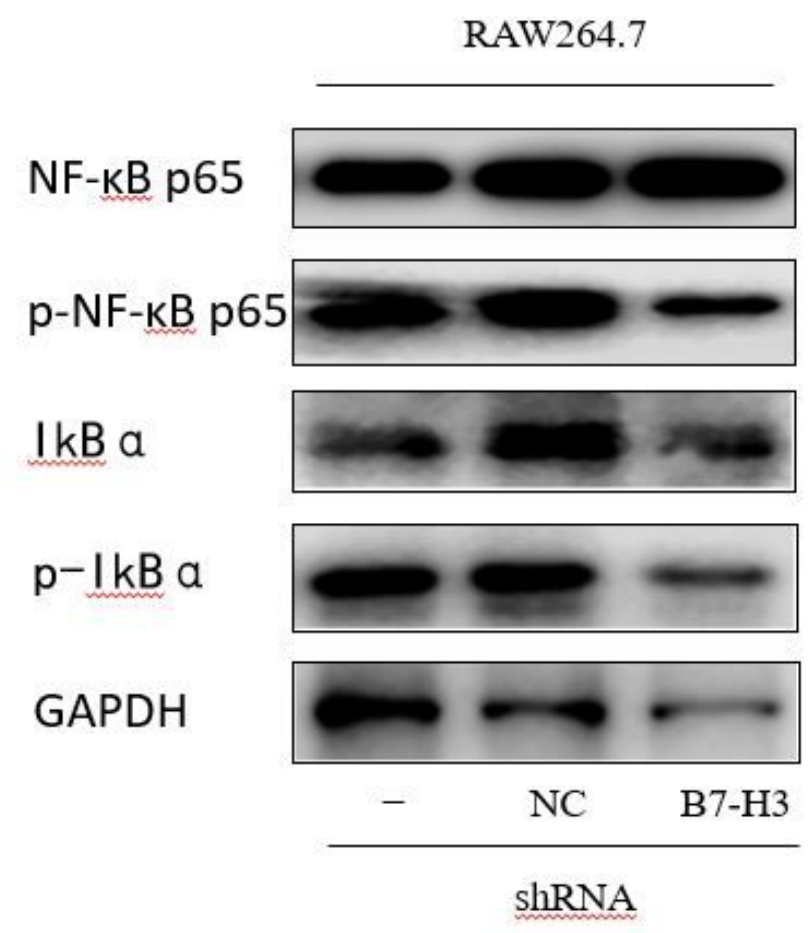

\section{Figure 7}

Effects of B7-H3 expression on NF-KB signaling in THP-1 and RAW264.7 cells. (A \& B) Levels of NF-KB p65, ІкBa, p-NF-кB p65, and p-IKBa were significantly reduced in (A) THP-1 cells transfected with LV3-hB7H3-1041 (hB7-H3-1041) compared to non-transfected cells (-) and cells transfected with negative control vector (NC) (B) RAW264.7 cells transfected with LV3-mB7-H3-294 (mB7-H3-294) compared to nontransfected cells $(-)$ and cells transfected with negative control vector (NC). All experiments were repeated for more than three times. 
A

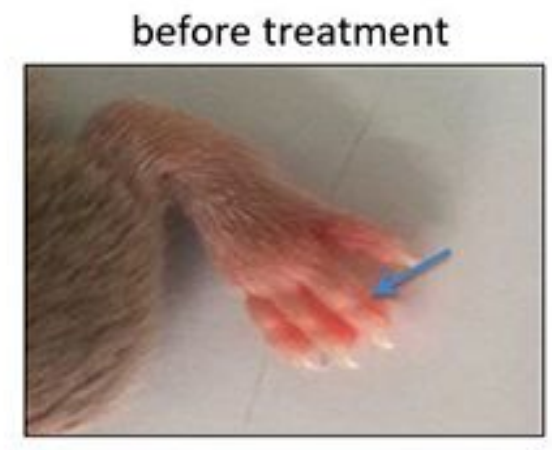

B

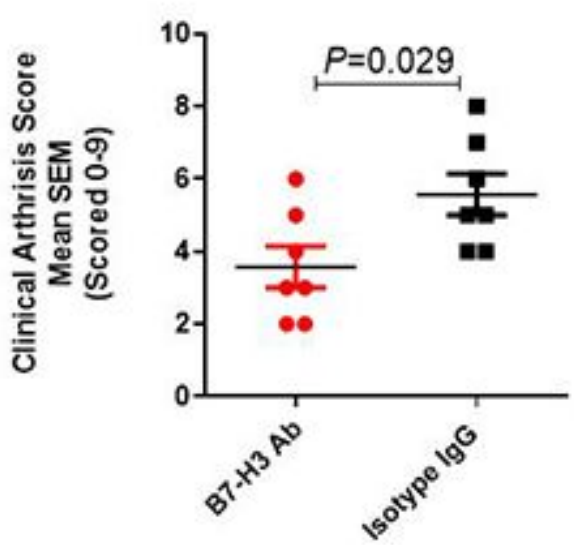

after treatment
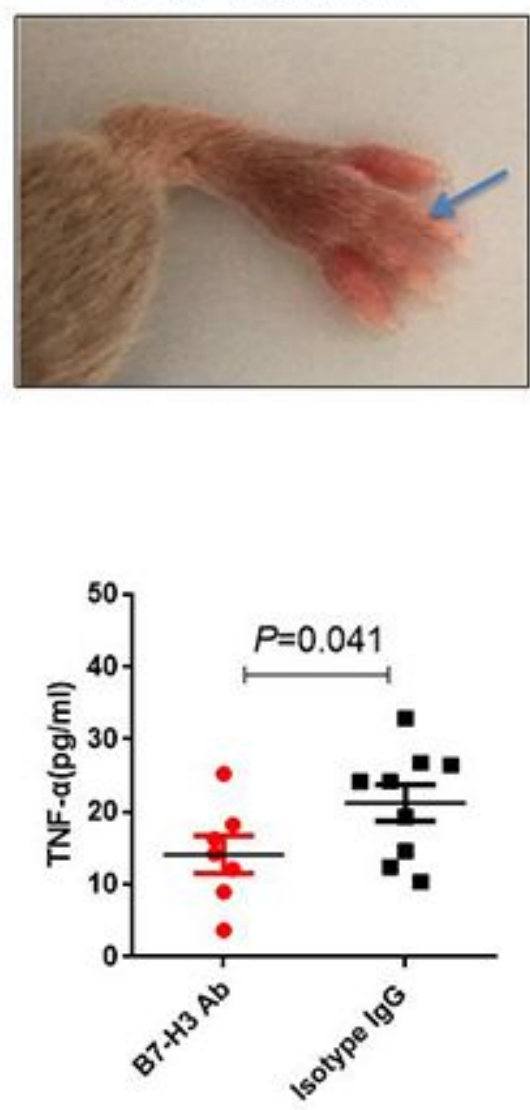
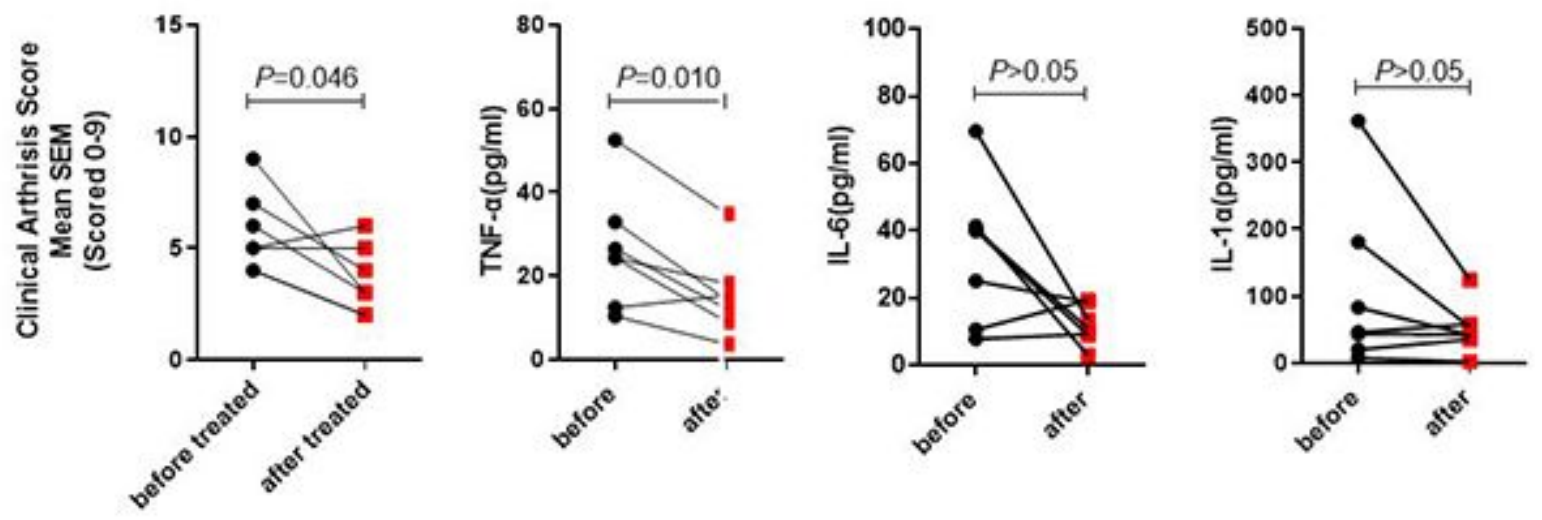

\section{Figure 8}

Anti-B7-H3 reduced the inflammatory response in CIA mice. (A) Representative image of a CIA mouse treated with anti-B7-H3. Anti-B7-H3 treatment significantly reduced inflammation (e.g., redness of toes). (B) Anti-B7-H3 significantly reduced the TNF-a level in peripheral blood after anti-B7-H3 treatment for 9 days. (C) Anti-B7-H3-treated DBA/1 mice induced with CIA for 9 days show a reduction in clinical arthritic score compared to isotype IgG control. (D) TNF-a, IL-6, and IL-1 levels in seven CIA mice before and after anti-B7-H3 treatment. $n=9 /$ normal group, $n=7-9$ /isotype IgG control and B7-H3 Ab groups. 